\title{
Measuring the Performance of Inventory Management System using Arena Simulator
}

\author{
Fawaz J. Alsolami \\ Computer Science Department \\ Faculty of Computing and Information Technology \\ King Abdulaziz University, Jeddah, Saudi Arabia
}

\begin{abstract}
The demonstration of inventory management systems is presented in this study to deal with a situation, where organizations are facing challenges due to uncertain behavior of demand. The implementation conducted using simulation technique to generate sampling experiment through computing and statistical methods. The important aspect of the simulation is to understand level of satisfaction regarding proposed system and its attributes. To assist the organization in controlling and managing the inventory system, the research provides the solution using Arena simulation tool. Firstly, the study explained the use of simulation and then reviews the common approaches of simulation. Secondly, the research proposed a framework for inventory control system. For practical implementation Arena tool used for model implementation. The main purpose of this experiment is to measure and analyze the applicability of potential system using a simulation tool. The results indicated the successful implementation of the proposed framework for inventory system. The model used multiple inventory system's variables such as demand, inventory stock and realized cost. For demonstrating the real world system's behavior, we used stochastic model for demand. The model executed using single server queuing (M/M/1) approach, but replicated several times. The results highlighted the high performances of machine located on multiples places and processed demand requests on each replication run. The study presented in this research demonstrates the association between demand and inventory. The proposed model can support the manufacturing organizations to control and manage inventory system.
\end{abstract}

Keywords-Inventory management system; simulation; arena simulation tool; demand and inventory

\section{INTRODUCTION}

Simulation is a method of imitating the real world scenario on a computing system using different technology and tools. Commonly, it is used for experiment and get sampling data using a computing techniques [1]. The main purpose of this research is to apply an efficient implementation of inventory management and control system using simulation strategy. The simulation analysis performed using time period factor, which is one of the important factors to realize the performance prior to implement the actual system. In addition, the simulation helps to create assumptions related to system and user's behavior, operational analysis, system effectiveness, and evaluation strategies [2].

Model plays an important role in performing simulation. As the variables defined inside the model provide real description of the system. Those variable are also known as measuring factors, which used for collecting sampling data from the selected population using a computer program [3]. With the help of the models the experiment can perform to dig out the probability of system implementation and behavior using different statistical and computing strategies [4]. Therefore, efficient model designing with all possible variables is the critical phase before simulation. Whereas, the testing and validation of models executed with the help of simulation. For this reason, most of the time the terminologies modeling and simulation are often used together [5]-[7], for the same purpose, to do the sampling experiment.

The general steps of applying simulation start from conceptual model $\rightarrow$ use of tool to verify the system $\rightarrow$ and finally validate the system as final output [8]. There are different factors and sub-tasks need to be performed on every step as shown in Fig. 1. The figure proposed earlier in the research showing the association between different phases during whole experiment. The first step in this figure is initiated by developing a conceptual model of an already implemented system that need to be enhanced or a new system to be developed. The second phase is to convert conceptual model into statistical, mathematical, or graphical framework. Third step is to implement the model using computing tool, which can generate statistical report for measuring the performance of the factors. Finally, the last phase is to validate the simulation performance using generated output. If the output is in the favor of the system it can be finalized, else the process will restart after proper modification in the conceptual model again. In this way, the trial and implement procedure applied by repeating the steps of implementation, which is the core of simulation.

Simulation is applied for many disciplines such as science, technology, health industry, manufacturing, education and others [2]. A quantitative analysis performed by implementing the supply chain management system using simulation strategies [9], [10]. The idea for controlling the inventory for agricultural product using simulation is presented by [11] using multiple attributes such as transportation cost, goods lost cost, and shortage cost. The research article suggested that the simulation tool can help in measuring the performance of inventory system and can assist the organization to develop the monitoring policy. The monitoring policy is used to create positive relationship between demand and supply variables. Overall, the process will further help the organization in building efficient inventory control system. 


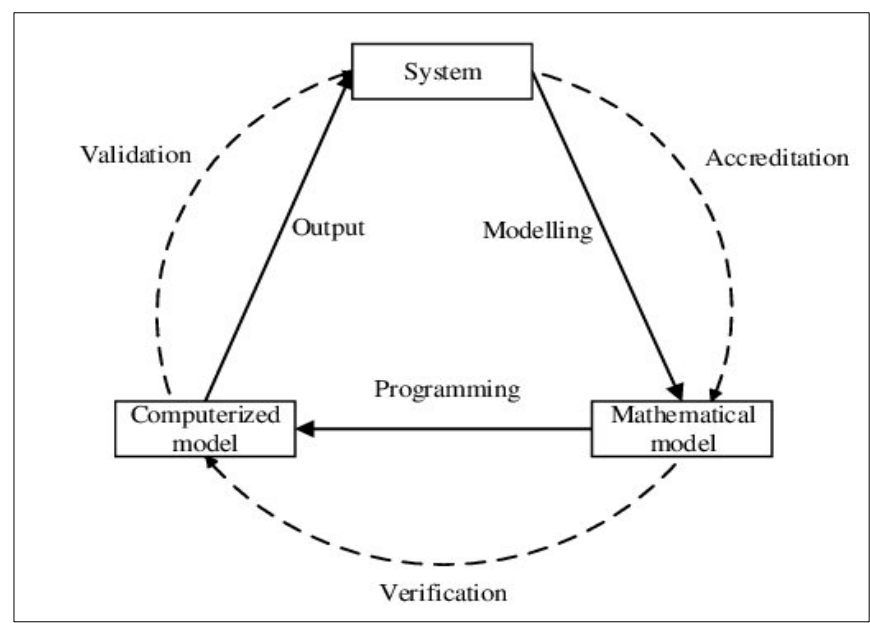

Fig. 1. Steps of Simulation [8].

The main problem has taken in this study is, "How an organization can improve and manage their inventory management system?” Therefore, the research proposes and validates the model for improving the inventory system using demand, supply and shortage variables. For this, the model validation applied using simulation tool, and to measure the performance of the model, replication applied several times. The research will help the organization to understand the behavior and impact of different variables of inventory system identified and analyzed in this research.

The rest of the paper is organized as follows. The next section discussed the research context of inventory control and management system. Section III described the differences between spreadsheet and Arena simulation. Afterwards, the proposed framework of inventory management system discussed in Section IV. Whereas, Section V illustrates the implementation of proposed model and results analysis generated by the simulation tool. Finally, the research ends by mentioning about final words and future work to enhance the research idea provided in this study.

\section{INVENTORY CONTROL MANAGEMENT SYSTEM - OVERVIEW AND RESEARCH CONTEXT}

Generally, Inventory Control and Management System (ICMS) has an important role in the organization, which integrated with different other system such as customer management system, marketing, and manufacturing. This kind of system is mainly responsible to deal with multiple operational activities, and it is one of the major requirements of the organization as the system contains detailed information of every product [12]. There are different attributes highlighted in previous research that handled by the ICMS. Modeling and simulation for ICMS applied and reviewed several time. There are different applications used for validating the model. Number of available products, demand and supply ratio, order and shortage cost, transportation and raw material cost are example of attributes used for ICMS model simulation [11].

Inventory management system can be a part of any organization dealing with customer and offering services. It usage in healthcare industry presented by [13] to improvise the services provided by the hospitals. This is one the critical organizations need to be operated their business processes without any delay and mistakes as dealing with different types of patients and lifesaving medicines. Inventory control of medicine and other surgical instruments are very essential to be managed properly. The study suggested major characteristics of inventory system operating in healthcare department. Some of the characteristics mentioned are, rapid and continuous changes in orders, use of medical accessories, availability of beds and surgeons, no definite time of patients to check out the hospital, and the dependency of medical accessories on each other.

Another approach of ICMS presented in the form of multilevel approach used by [11] to deal the problem of agricultural products. The purpose of that system was to control the request in different layers to improve the overall performance of ICMS. The simulation results showed that the multilevel hierarchy helped them to deal with technical issues and to develop inventory control policy. The hierarchy presented in Fig. 2, showing the association between suppliers, manufacturers, retailers, and customers in ICMS. The hierarchy is in generic format, which can be useful for any industry. But the important thing in this approach is the development of communication channels to control the inventory system. The journal of simulation published an article that proposed the hybrid approach improving the performance of inventory system [14]. The article suggested number of important factor during simulation of ICMS that needs to be taken under consideration during ICMS development. Those factors are; uncertainty in demand and supply, the ratio of returned items, and the association between manufacturing and remanufacturing. The algorithm used in that study was Mixed-Integer Linear Programming.

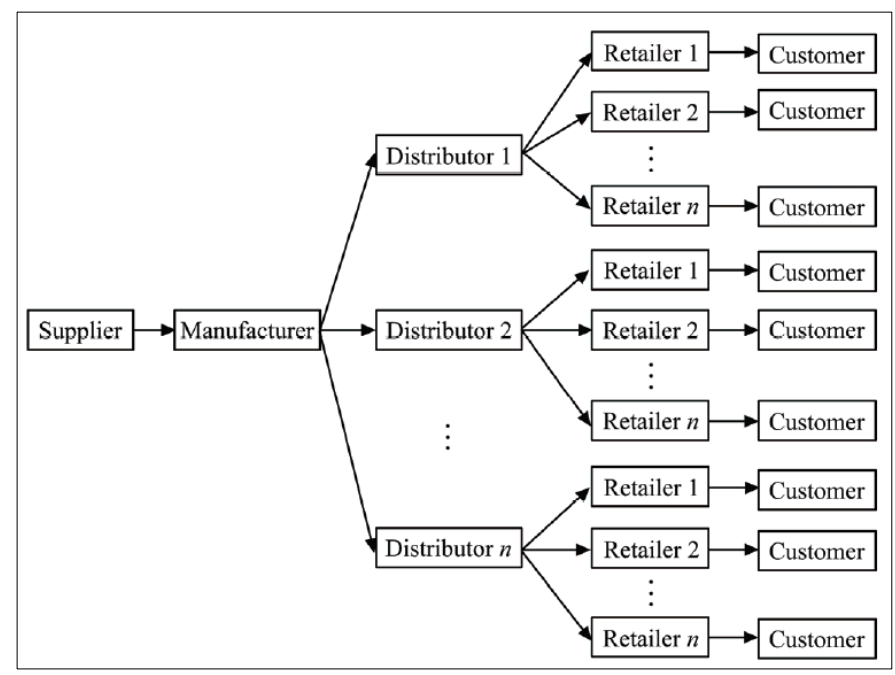

Fig. 2. The Working Scenario of ICMS [11].

Furthermore, the same kind of idea presented that described the level of uncertainty in producing the quantity and maintaining the quality of the products [15]. The proposed model was developed for the concept of hybrid manufacturing system. As previous literature suggested that inventory models are using two different types of approaches; Deterministic and Stochastic. The first one is used for the model where all 
variables are known with certainty. Whereas the second one is representing where demand and products involved uncertainty [16]. In order to cover up the uncertainty and matching with demand and supply, the research presented the idea for adding a layer of multiple supplier [17]. The simulation strategy was applied to validate the propose model, which also highlights the use of simulation technique for validating the ICMS model.

The latest technology has already changed the working environment in every organization. Internet of Things (IoT) [12], cloud computing [18], and big data [19] are some current development in computing field that allows the organization to enhance their information technology infrastructure. Accordingly, taking the advantage of current technologies [20] proposed the idea of automating the inventory process applied in warehouses. The purpose was to reduce the human efforts and automate the whole process. In addition, the forecasting models for inventory system suggested in a research by combining the forecasting parameters with inventory policy metrics [21]. The inventory models can work using batch approach. The batch approach means, where the demand received in a bunch. A research discussed this scenario where they check the execution of this approach using two models known as single server and multi-server [22]. In addition, the advanced version of moving average method applied in the research to implement the idea of downstream interface for supply chain management by reducing inventory levels [23]. That research is very new and the journal just published preproof for that research yet, which shows the importance and newness in the research idea undertaken in this study. Another latest research conducted the simulation of inventory management on a real vending network system [24]. The model used stochastic approach for dealing with demand attribute, which is one of the major constraints in inventory system.

This section discussed and put stressed on presenting the comprehensive overview and research context of ICMS. The inventory system is widely used for controlling the product's in and out records in the organization. Regarding ICMS, the following assumptions are extracted from the literature review:

- ICMS is one of major components in almost every organization.

- The major attributes of ICMS are demand, order, supply, shortage, product's cost, and availability of the products.

- Deterministic and Stochastic are two kinds of model used for dealing with demands.

- The major limitation founds in ICMS are uncertainty of demand and orders, cost of products, seasonal purchasing, product's return, and risk involvement in each process.

- Simulation is very commonly applied for improving the ICMS.

- The major tool has been used for ICMS simulation are, Spreadsheet Simulation and Arena.

\section{Simulation TOOLS}

Simulation is used for doing an experiment based on abstract model, to analyze, measure, and understand its real implementation [25]. It has many phases to execute this process, started from building a conceptual model using different kinds of variables. The variables can be independent, mediating, and dependent based on the requirements of the system. Whereas, the dependent variable is also known as decision variable, which directly associated with independent variables [26]. This section describes the common tools used for simulation experiment as follows.

\section{A. Simulation using Spreadsheet}

Spreadsheet is one of the traditional program used for various purposes. First time, the concept of spreadsheet initiated by VisiCalc in 1979, which had restricted functionalities but the idea was prominent that further leads to progressive development till now [27]. The latest version of Microsoft Excel has the efficacy to collaborate with different other applications such as visual basic, statistical tool, simulation, decision support system and others [28]. The idea of spreadsheet simulation executed by [3] where they performed the implementation using inventory management system's example. In addition, there are other published work as well discussed and used the concept of spreadsheet simulation [29], [30]. A graphical representation of spreadsheet simulation is shown in Fig. 3. There are number of factors and characteristics highlighted for spreadsheet tool related to simulation as discussed below [3].

- It provides flexible way to initiate variables and create association between the variables.

- The strong formula and equation development option is there to use and generate results.

- Generating of random number and other number distribution option can facilitate to perform simulation.

- Use of data table to recreate the situation and repeat the experiment multiple times.

- The visualization techniques are there to illustrate the input, process and output procedures.

- Easy access to external application using add-in option.

- Overall, automated, integration, and flexible environment.

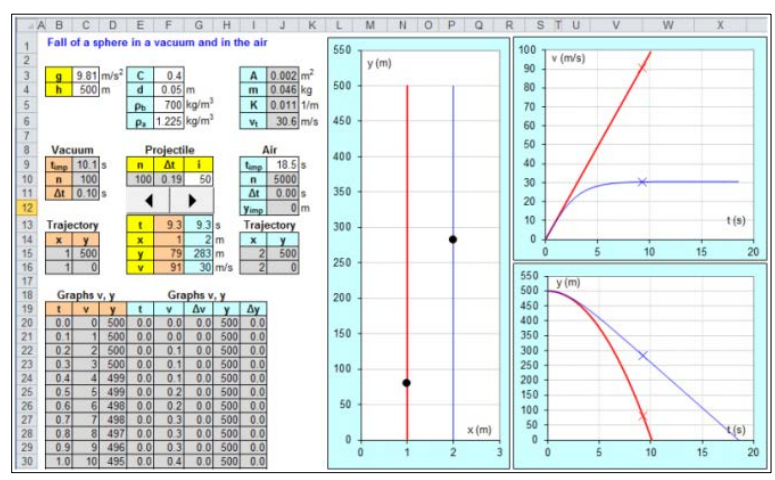

Fig. 3. Simulation Experiment using Spreadsheet [31]. 
Apart from simulation, there are many additional operations such as decision support system, programming facilities, data organization, statistical analysis and others are available in excel. The list of major functionalities provided by spreadsheet, but not limited provided by [32] as defined below.

- There are big number of built-in functions available in excel such as financial, statistical, pivot table, database.

- Easy and integrated tool for visualizing the dataset using graphs and charts options.

- The facility for linear programming, visual basic programming, and mathematical programming.

- The tool has the ability to integrate with different tool like risk analysis, sensitivity analysis, decision analysis and others.

\section{B. Simulation using Arena}

Nowadays, a latest tool using for simulation by many industries is known as Arena that has been designed by Rockwell Software, Microsoft [33]. The tool is common for conducting experiment and collecting samples to estimate the chances of the proposed system's implementation. The major industries assisting help from this tool are many such as hospitals, inventory systems, order delivery systems, queue estimating process and others [34]. It provide the flexible environment where all of the operators are readily available and just required to connect them during process development. It also has the facility to develop model and transform in Arena, where the model can easily convert in its environment [4].

The tool has widely used in previous researches, defining the high applicability and use of the tool in academics and industries. Development of inventory control model with all specifications in manufacturing industry applied [14] to analyze and simulate the proposed model. Another research presented the common ideas can be applied using this tool. The research suggested the tool can be useful for the industries such as manufacturing, supply chain, and inventory system [35]. That article presented various models development strategies using Arena. Product manufacturing, consumer market, market demand, and component ordering simulation model presented with all possible steps.

The working environment in Arena has divided in two main layers called "Flowchart" and "Spreadsheet". There is a panel in the tool describing all available processes under the categories of "Basic" and "Advanced". The process development is easy to handle as there is no programming involve. The list of operator can be used by drag and drop, where the further specifications can be filled by double click on the operator. Mainly, the process development in this tool used the same programing strategy and connected as input $\rightarrow$ process $\rightarrow$ output. Following are the major characteristics provided by this tool.

- It provides the way to generate number of variables with all parameters.
- It offers to create different types of resources such as machine, materials, and components.

- The entities queue can be created for resources use.

- The entities can arrive as one by one or in batch.

- Different statistical methods can apply for generating number distribution.

- The option is available to define own expression for calculating and building user's own formula.

\section{ICMS SIMULATION - PROPOSED FRAMEWORK}

To support the idea presented in this research, this section proposed a framework for ICMS simulation as shown in Fig. 4. The idea of this framework is based on the inventory model presented and simulated using spreadsheet tool [3]. The original idea was applied in spreadsheet simulation, while this research implemented the simulation using Arena tool. The inventory model example used in this study is associated with sport club players. Every day, number of players are presenting in the club for different activities. Based on the experience data, it has been found that most of time the demand is either got short or excess based on the attendance.

Due to this fact the sports club management always got in trouble for not fulfilling the requirements of players. Moreover, the shortage and excess of demand cause them financial and other loss. The proposed model shown in Fig. 4 illustrating the simulation approach will be applied in this study using Arena. There are six major phases mentioned in the model. The definition of each phase is defined below, whereas the actual list of variables and values applied in Arena is described in the next section.

1) Variable Identification: This phase is to identify number of variables to be used in the simulation. It mainly depends on system's specification.

2) Input Values to the Variables: This research used the demand variable as stochastic, where the distribution will be known but uncertainty will be involved to make it dynamic variable. For other variables can be taken as dynamic or fixed.

3) Computation for each Variable: The calculation of each variable using a prescribed formulae and notation. This calculation is prior to simulation, means if any variable required any extra computation or assignment.

4) Calculation if Demand is More or Less: What happen if demand is less or more? Here we will use a decision statement for both conditions. But the calculation will be different. It will help to compute loss for both conditions.

5) Run Simulation and Replication: But in both ways simulation will run. For more clear understanding we can replicate the simulation several times.

6) Result Analysis: The result can show the impact of shortage and excess in demand. It will show the financial loss due to not managing the inventory system properly. The result will be displayed using statistical and visualization tools. 


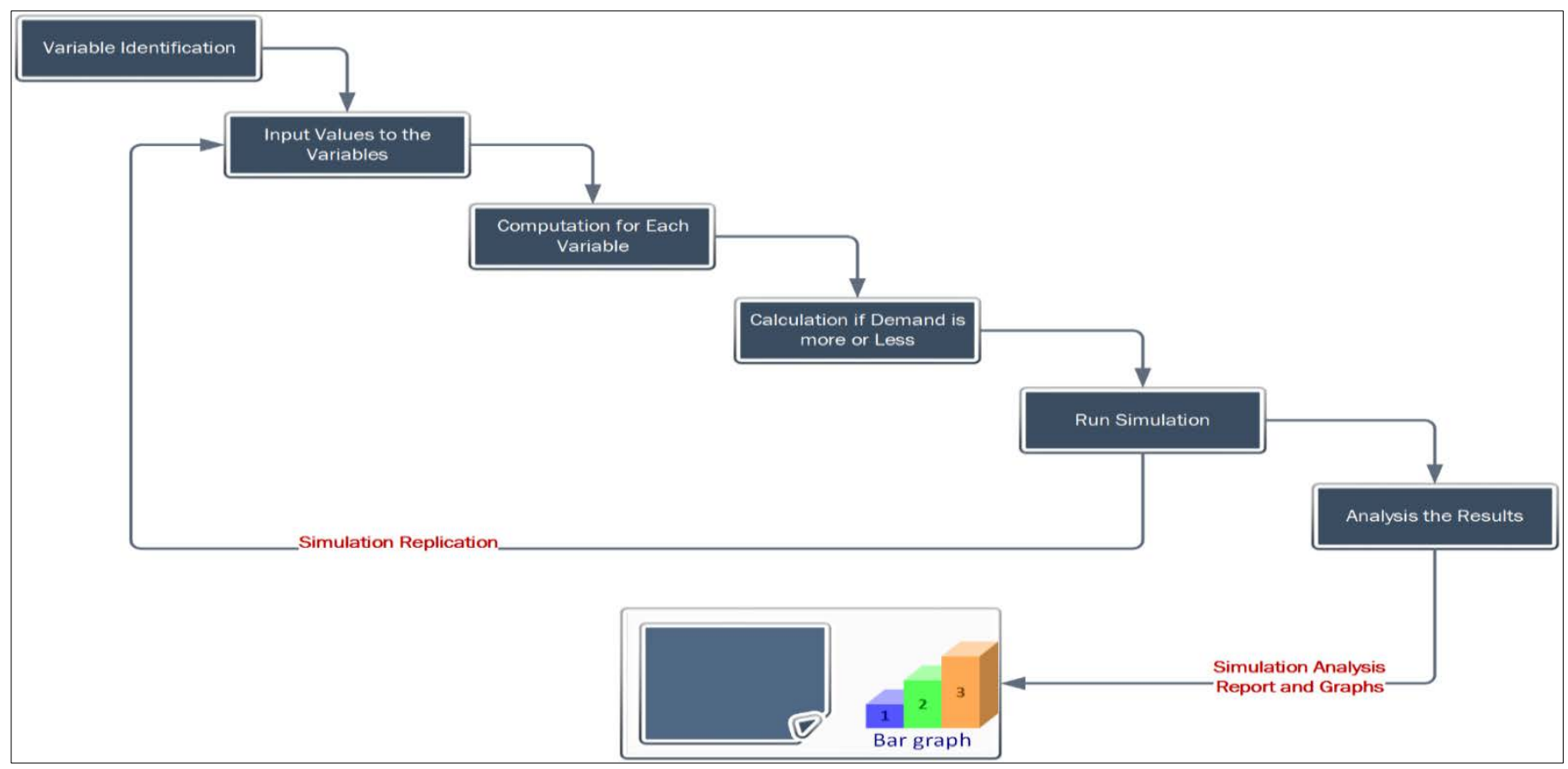

Fig. 4. Proposed Model for Inventory System Simulation.

\section{FRAMEWORK IMPLEMENTATION USING ARENA}

The main research problem undertaken in this study is to support organization by developing inventory control model using dynamic values of demands. Another aspect of this study, to automate the overall process of inventory control and management system. Therefore, simulation technique used to test and validate the model. The Arena software is used for implementation of the model. Normally, the model should have quantitative or qualitative values associated with one or many attributes identifying the operational work of the proposed system. Finally, the output of the experiment can highlight the strength and weaknesses of the proposed system. The output can lead the organization to analyze the probability of system implementation in real world. The subsequent section is defined the model specification, variable identification, model implementation, and result analysis.

\section{A. Inventory Model - Variable Identification}

Mainly, the inventory model based on the variable like demand generates by the customer. The model considered in this study is based on controlling the inventory of a sport club. The scenario of club is, there are many registered club members visiting club to do sports activities. One of the services provided by the club is to offer them food on every visit. The main complexity in arranging the food is the unknown number of visitors. Therefore, to solve this issue they need a system which can control and manage their food arrangement inventory system. The same problem is implemented using spreadsheet simulation, while in this study we proposed a model to be implemented in Arena. The main problem in this scenario is the financial loss because of not matching the actual demand and items availability. The number of variable used in this model illustrated in Table I. There are two different situations that organization is facing within this problem also mentioned in this table.
TABLE I. VARIABLES SPECIFICATION

\begin{tabular}{|c|c|}
\hline Variable & Specification \\
\hline Players Arrival & $\begin{array}{l}\text { Entities per Arrival: } 10 \text { (Batch) } \\
\text { Distribution: Random } \\
\text { Simulation Time: } 1 \text { hour }\end{array}$ \\
\hline Players Demand & $\begin{array}{l}\text { Assign: Demand and inventory stock variable } \\
\text { Initiation } \\
\text { Distribution: Random }\end{array}$ \\
\hline Check Inventory? & $\begin{array}{l}\text { Decision Box: Check if inventory has the available } \\
\text { demand? }\end{array}$ \\
\hline $\begin{array}{l}\text { If Demand Excess than } \\
\text { Inventory, Lost? }\end{array}$ & Computation: It will cost $\$ 60$ per item \\
\hline $\begin{array}{l}\text { If Demand Shortage } \\
\text { than Inventory, Lost? }\end{array}$ & Computation: It will cost $\$ 160$ per item \\
\hline $\begin{array}{l}\text { Reprocess and Record } \\
\text { Lost Amount for } \\
\text { Excess }\end{array}$ & $\begin{array}{l}\text { Reprocess: by putting another resource machine } \\
\text { for recovery. } \\
\text { Record: the total amount lost for excess demand. }\end{array}$ \\
\hline $\begin{array}{l}\text { Reprocess and Record } \\
\text { Lost Amount for } \\
\text { Shortage }\end{array}$ & $\begin{array}{l}\text { Reprocess: by putting another resource machine } \\
\text { for recovery. } \\
\text { Record: the total amount lost for shortage } \\
\text { demand. }\end{array}$ \\
\hline
\end{tabular}

\section{B. Model Implementation and Result Discussion}

As shown in the Fig. 5, the simulation started after the players arriving to the premises in random number. The arrival is in batch, means group of 10 members arriving in every event. The simulation run for 1 hour and replicated for 10 times. The arrival of the players, by default create the variable "order", which shows that each players will required a meal box. The next step in the simulation is the assignment of variable "demand" using player's arrival information. Therefore, the demand variable will be created here same as number of players arriving after prescribed time using random distribution. 
The purpose of this assignment is that demand variable will be used at different location in later simulation step. Meanwhile, at the same step another variable will be initiated called "inventory" stock available. It will further validate the difference between demand and stock availability. The processing of players orders then execute based on the availability of machine (resource) provided at next step known as "process". There will be a different processing time for each order. The working time averages is discussed in further result section. Therefore, the processing machine will use some time-delay in processing the orders. The next step is the decision box for validating the demand value with inventory stock. Here the condition applied that "if inventory is greater and equal to Demand". If the statement is "true" it will go to "demand excess" node. The decision box implementation is the fourth step in this simulation.

Moving forward, the next step is to reprocess the simulation in both condition, either demand is less or more. Adding a machine over here is indicating that the additional time is required to process the request for both scenario. The final step in this simulation to record the different variables executed in this simulation. At last, we run the simulation 10 times to analyze the different situations, calculate the averages, and check the average lost in 10 simulations.

The results indicates the expected cost computed for both situation, if demand is less than the inventory, or if demand is more than the inventory. As discussed earlier, the spreadsheet simulation applied earlier by [3], is used in this study to apply the simulation using Arena. Therefore, the result generated based on the following modified equation.

$z=c_{e}(y-D)$ if $y \geq D$

If inventory $(y)$ is greater than equal to the demand $(D)$. It represents that there will be excess cost $\left(C_{e}\right)$ applied, to calculate the realized cost $(z)$. In this scenario, the realized cost, will be computed by subtracting the inventory from demand, then multiply it by cost of excess that is $\$ 60$ as mentioned in Table I. We calculated the values for each simulation to know the realized cost in all simulation, is shown in Table II. The next formula applied is for computing the shortage cost as described below.

$z=c_{S}(D-y)$ if $y<D$

If inventory (y) is less than to the demand (D). It represents that there will be shortage cost (Cs) applied, to calculate the realized cost $(\mathrm{z})$. In this scenario, the realized cost, will be computed by subtracting the demand from inventory, then multiply it by cost of shortage that is $\$ 160$ as mentioned in Table I. We calculated the values for each simulation to know the realized cost in all simulation, is shown in Table II.

After all discussion, and how the values are computed to understand the financial loss using a sample experiment conducted in this study. The table representing the summary of simulation, which denotes the samples collected and realized cost. The objective of this simulation is to find out the optimal association between demand and inventory stocks. Searching of ideal values cannot generate by single run, therefore, the experiment conducted several times to create multiple values and analyze the results. The experiment used a sample cost in each replication to calculate the shortage and excess found in inventory and demand. The purpose was to idealize the best scenario and association between both variables. One of the screenshots of using the variables in the model and assigning some input values is shown in Fig. 6.

It can be evident from Table II that out of 10 simulation run, replication number 4 gives the lowest financial lost (60\$), whereas replication number 10 provides the maximum lost to the sports club that is $11680 \$$. Another ideology to be considered from this result is, if inventory is more than the demand the lost amount is less. On the other side, if inventory is less than demand the lost amount is high. Based on the result generated, the sports club can create a strategy, to keep the inventory stock as high as they can do, as the financial loss is minor in this situation. Although, the experiment used a sample of data generated randomly, but the idea was to validate the inventory model for sport club, supported by different variables.

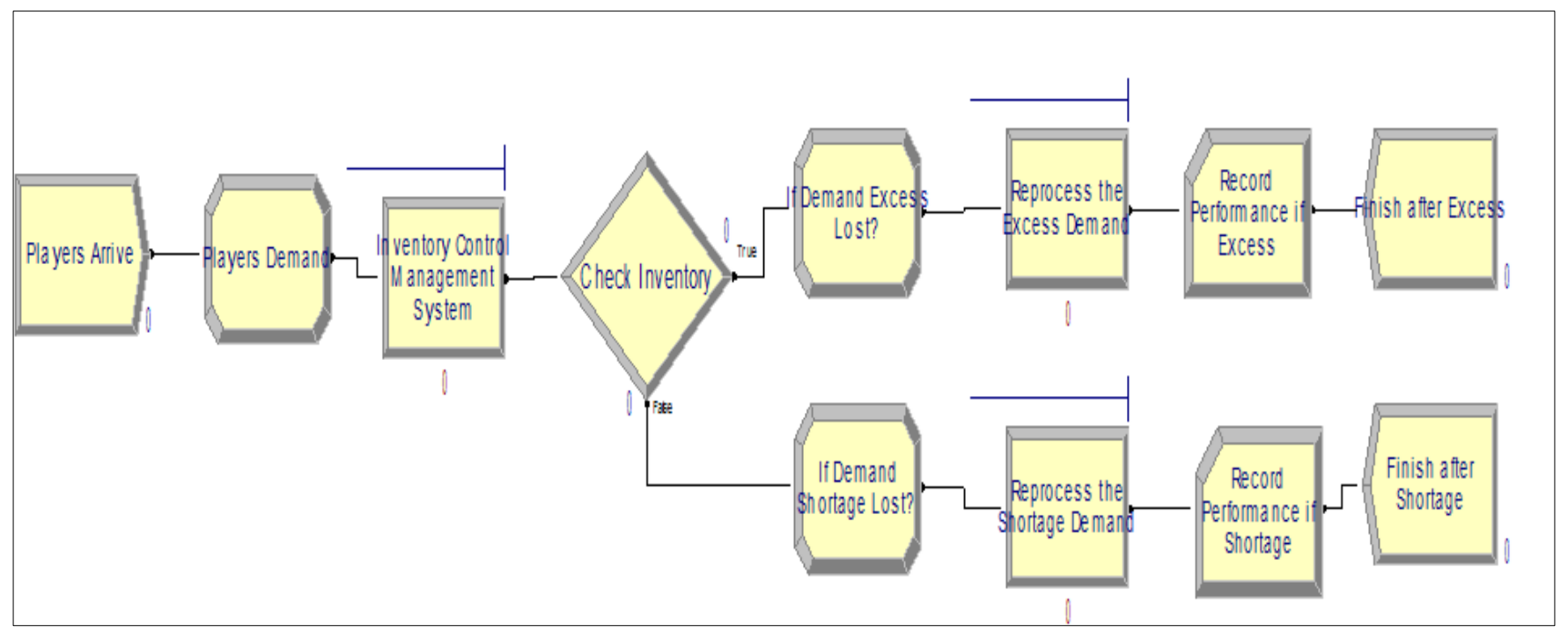

Fig. 5. Screenshot of Model Implementation in Arena Simulator. 


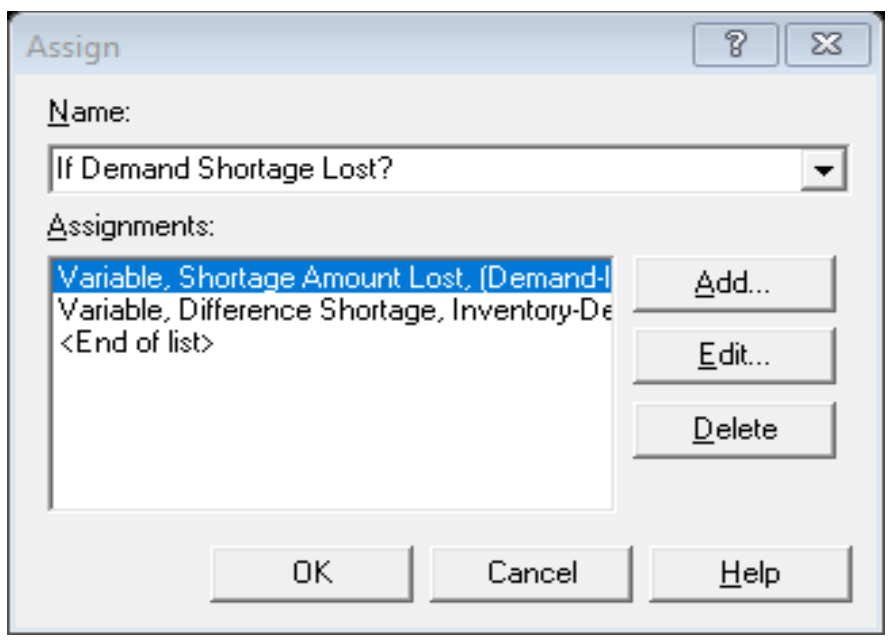

Fig. 6. Input and Assignment to the Variables.

TABLE II. SIMULATION RESULTS

\begin{tabular}{|l|l|l|l|}
\hline Replication \# & Demand & Inventory & Realized Cost \\
\hline 1 & 57 & 13 & $7040 \$$ \\
\hline 2 & 24 & 63 & $2340 \$$ \\
\hline 3 & 26 & 52 & $1560 \$$ \\
\hline 4 & 84 & 85 & $60 \$$ \\
\hline 5 & 40 & 48 & $480 \$$ \\
\hline 6 & 53 & 56 & $180 \$$ \\
\hline 7 & 40 & 2 & $6080 \$$ \\
\hline 8 & 26 & 47 & $1260 \$$ \\
\hline 9 & 59 & 12 & $7520 \$$ \\
\hline 10 & 95 & 22 & $11680 \$$ \\
\hline Mean Values & $\mathbf{5 0 . 4}$ & $\mathbf{4 0}$ & $\mathbf{2 6 4 4} \$$ \\
\hline
\end{tabular}

In addition, the mean values generated in the simulation indicating that, overall the demand value is higher than the inventory stock. In 10 replications, the mean value of customer's demand recorded as 50.4, and store stock are 40, while the average lost recorded is $2644 \$$. The number of replication is few in this experiment but based on the result, it can be suggested to the organization to increase the inventory stock to match it with the demand. Although, it should be clear that if there is a difference in stock and demand, it will always damage the organization reputation.

Finally, to understand the different machine's performance at each station, Table III is representing the details of each machine. In this table, the recorded time is representing the average working time during all replications. It can be seen that, the average working time is recorded at "inventory control system" is the highest, as this was the first machine and all requests received at this station. After that the request was distributing and forwarding to two different nodes. Like in some replication it went towards the "Excess Demand Reprocess" or sometime forwarded to "Shortage Demand Reprocess". Therefore, their working time is considerably lower than "inventory control machine".
TABLE III. OVERALL WORKING TIME AT EACH STATION

\begin{tabular}{|l|l|l|l|}
\hline Station & $\begin{array}{l}\text { Overall } \\
\text { Average } \\
\text { Values }\end{array}$ & $\begin{array}{l}\text { Overall } \\
\text { Minimum } \\
\text { Values }\end{array}$ & $\begin{array}{l}\text { Overall } \\
\text { Maximum } \\
\text { Values }\end{array}$ \\
\hline Inventory Control System & 0.87 minutes & 0.36 minutes & 0.90 minutes \\
\hline $\begin{array}{l}\text { Reprocess the Excess } \\
\text { Demand }\end{array}$ & 0.085 minutes & 0.05 minutes & 0.11 minutes \\
\hline $\begin{array}{l}\text { Reporcess the Shortage } \\
\text { Demand }\end{array}$ & 0.074 minutes & 0.03 minutes & 0.13 minutes \\
\hline
\end{tabular}

\section{CONCLUSION}

The research proposed a model to support organization in dealing with inventory control and management system. The idea is useful for those organizations to take decision based on the generated results. The implementation of proposed model executed with the help of simulation strategy. Specifically, the model used stochastic model of demand, where the variable were uncertain and selected randomly in each replication. The inventory optimization is supposed to be a complex situation, where all organizations accepting unknown damage every time. The research is proposed a model with limited variables to support this idea, which can be enhanced by adding more variables, machines, and stations. The result of the simulation guided that availability of stock will cause less damage, while having lower stock than demand is always giving lost to the organization. It will not only cause the financial lost but it will also degrade the company's reputation.

\section{FUTURE WORK}

The model can be improved using demand and supply variables with multi-server strategy. In this study, the researcher used the single server model, where the multiple of entities served with one machine. The idea can be amended by implementing multiple server approach on different scenario. The multiple server approach can provide the analysis and performance of different machine, which can also be used to compare the performances of different machines. The simulation results can provide the statistics to the organization in order to take the decision based on the performance of the model.

\section{REFERENCE}

[1] G. S. Fishman, Monte carlo concepts, algorithms and applications. New York: Springer, 1996.

[2] R. D. Smith, "Simulation," Encycl. Comput. Sci., 2000.

[3] A. F. Seila, "SPREADSHEET SIMULATION," in Proceedings of the 2006 Winter Simulation Conference, 2006, pp. 11-18.

[4] Y. Merkuryev and A. Grinbergs, "Design of UML models and their simulation using ARENA," WSEAS Trans. Comput. Res., vol. 3, no. 1, pp. 67-73, 2008.

[5] H.-J. Bungartz, S. Zimmer, M. Buchholz, and D. Pflüger, Modeling and Simulation: An Application-Oriented Introduction. Springer-Verlag Berlin Heidelberg, 2014.

[6] S. Raczynski, Modeling and Simulation: The Computer Science of Illusion. Wiley Online Library, 2006.

[7] B. Zeigler, A. Muzy, and E. Kofman, Theory of Modeling and Simulation, 3rd ed. Elsevier, 2018.

[8] D. Gao, X. Xu, J. Yin, H. Zhang, and B. Zhang, "SDG and qualitative trend based model multiple scale validation," in IOP Conference Series: Materials Science and Engineering, 2017, vol. 231, no. 1.

[9] A. J. Schmitt and M. Singh, "A quantitative analysis of disruption risk in 
a multi-echelon supply chain,” Int. J. Prod. Econ., vol. 139, no. 1, pp. 22-32, 2012.

[10] T. Peirleitner, A. J., Altendorfer, K., \& Felberbauer, "A simulation approach for multi-stage supply chain optimization to analyze real world transportation effects.," in 2016 Winter Simulation Conference (WSC), 2016, pp. 2272-2283.

[11] G. Xu, J. Feng, F. Chen, H. Wang, and Z. Wang, "Simulation-based optimization of control policy on multi-echelon inventory system for fresh agricultural products,” Int. J. Agric. Biol. Eng., vol. 12, no. 2, pp. 184-194, 2019.

[12] B. S. S. Tejesh and S. Neeraja, "Warehouse inventory management system using IoT and open source framework," Alexandria Eng. J., vol. 57, no. 4, pp. 3817-3823, 2018.

[13] E. Saha and P. K. Ray, "Modelling and analysis of inventory management systems in healthcare: A review and reflections," Comput. Ind. Eng., p. 106051, 2019.

[14] P. Thammatadatrakul and N. Chiadamrong, "Optimal inventory control policy of a hybrid manufacturing-remanufacturing system using a hybrid simulation optimisation algorithm,” J. Simul., vol. 13, no. 1, pp. 14-27, 2019.

[15] H. Rachih, F. Z. Mhada, and R. Chiheb, "Simulation of a stochastic inventory model for a hybrid manufacturing-remanufacturing system," in 2019 International Colloquium on Logistics and Supply Chain Management (LOGISTIQUA), 2019, pp. 1-6.

[16] A. E. E. J. F. Moritz, J. M. Bloemhof-Ruwaard, V. der Laan, E. Van Nunen and L. N. Van Wassenhove, "Quantitative models for reverse logistics: A review,” Eur. J. Oper. Res., vol. 103, no. 1, 1997.

[17] A. Bartoszewicz and P. Latosiński, "Sliding mode control of inventory management systems with bounded batch size," Appl. Math. Model., vol. 66, pp. 296-304, 2019.

[18] A. Elragal and M. Haddara, "The Future of ERP Systems: look backward before moving forward," Procedia Technol., vol. 5, no. 2212, pp. 21-30, 2012.

[19] A. L’Heureux, K. Grolinger, H. El Yamany, and M. Capretz, "Machine Learning with Big Data: Challenges and Approaches," IEEE Access, 2017.

[20] A. M. Vamsi, P. Deepalakshmi, P. Nagaraj, A. Awasthi, and A. Raj, "IOT Based Autonomous Inventory Management for Warehouses," in EAI International Conference on Big Data Innovation for Sustainable Cognitive Computing, 2020, pp. 371-376.
[21] N. Kourentzes, J. R. Trapero, and D. K. Barrow, "Optimising forecasting models for inventory planning,” Int. J. Prod. Econ., p. 107597, 2019.

[22] S. R. Chakravarthy and A. Rumyantsev, "Analytical and simulation studies of queueing-inventory models with MAP demands in batches and positive phase type services," Simul. Model. Pract. Theory, p. 102092, 2020.

[23] Y. Tliche, A. Taghipour, and B. Canel-Depitre, "An Improved Forecasting Approach to Reduce Inventory Levels in Decentralized Supply Chains,” Eur. J. Oper. Res., vol. Journal Pr, no. Pre-Proof, 2020.

[24] H. Grzybowska, B. Kerferd, C. Gretton, and S. T. Waller, "A simulation-optimisation genetic algorithm approach to product allocation in vending machine systems," Expert Syst. Appl., vol. 145, p. 113110, 2020.

[25] C. F. Cheung, C. M. Cheung, and S. K. Kwok, "A knowledge-based customization system for supply chain integration,” Expert Syst. Appl., vol. 39, no. 4, pp. 3906-3924, 2012.

[26] E. Turban, R. Sharda, and D. Delen, Decision Support and Business Intelligence Systems, 10th ed. Pearson, 2010.

[27] D. Cassel, "How VisiCalc's Spreadsheets Changed the World," The New Stack, 2019.

[28] Microsoft, "Microsoft Excel.".

[29] W. L. Winston, "Simulation modeling using @risk," Belmont, California: Duxbury, 1996.

[30] A. F. Seila, V. Ceric, and P. Tadikamalla, "Applied simulation modeling,” Belmont, California: Brooks-Cole, 2003.

[31] J. Benacka, "Numerical modelling with spreadsheets as a means to promote STEM to high school students," EURASIA J. Math. Sci. Technol. Educ., vol. 12, no. 4, pp. 947-964, 2016.

[32] C. T. Ragsdale, Spreadsheet modeling and decision analysis. Thomson south-western, 2004.

[33] R. S. Inc, “Arena User’s Guide,” USA, 2005.

[34] “Arena Simulation Software.” 2000.

[35] G. E. Vieira, "Ideas for modeling and simulation of supply chains with arena," in Proceedings of the 2004 Winter Simulation Conference, 2004. 